EFI 97-33-Rev

Revised January 1998

hep-ph/9707473

\title{
IMPROVED TESTS OF RELATIONS FOR BARYON ISOMULTIPLET SPLITTINGS 1
}

\author{
Jonathan L. Rosner \\ Enrico Fermi Institute and Department of Physics, University of Chicago \\ 5640 S. Ellis Avenue, Chicago IL 60615
}

\begin{abstract}
The least well-known octet baryon mass is $M_{\Xi^{0}}=1314.9 \pm 0.6 \mathrm{MeV}$. The prospect of an improved measurement of its mass by the $\mathrm{KTeV}$ experimental program at Fermilab, and opportunities for improvements in charged and excited hyperon and $\Delta$ mass measurements, makes it timely to re-examine descriptions of isospin splittings in baryons containing light quarks. By examining such relations as the Coleman-Glashow relation $M_{n}-M_{p}+M_{\Xi^{-}}-M_{\Xi^{0}}=M_{\Sigma^{-}}-M_{\Sigma^{+}}$one can distinguish between those models making use of one- or two-body effects involving quarks and those involving genuine three-body effects. A hierarchy based on an expansion in $1 / N_{c}$, where $N_{c}$ is the number of quark colors, is useful in this respect. The present status of other quark-model mass relations involving $\Lambda-\Sigma^{0}$ mixing and the baryon decuplet is also noted, and the degree to which one can determine parameters such as quark mass differences and individual electromagnetic contributions to splittings is discussed.
\end{abstract}

\footnotetext{
${ }^{1}$ Revised version submitted to Phys. Rev. D.
} 


\section{INTRODUCTION}

The electromagnetic mass splittings of the baryons in the flavor octet of $\mathrm{SU}(3)$ can be understood as a result of several effects in the quark model. (a) The $u$ and $d$ quarks have different masses, affecting both static and kinetic energies. (b) The quarks in a baryon have pairwise Coulomb interactions. (c) The strong hyperfine splittings (understood after the advent of quantum chromodynamics (QCD) as being due to the chromomagnetic interactions between quarks) can differ as a result of different $u$ and $d$ masses. (d) Electromagnetic hyperfine interactions between quarks are present. A sample of the post-QCD literature, from which earlier observations can be traced, is contained in Ref. [1].

Remarkably, there exist plausible limits in which all these effects preserve one linear relation among the masses of the baryon octet of flavor $\mathrm{SU}(3)$ [2]:

$$
M_{n}-M_{p}+M_{\Xi^{-}}-M_{\Xi^{0}}=M_{\Sigma_{-}}-M_{\Sigma^{+}}
$$

despite substantial symmetry-breaking effects in quark masses. The resistance of Eq. (II) to symmetry violations was pointed out in Ref. ([3]), and has been noted recently by Jenkins and Lebed in the context of a $1 / N_{c}$ expansion [4, where $N_{c}$ is the number of colors in QCD. (See also the later study by Bedaque and Luty [5].) In the present work we discuss the status and future prospects for testing this relation, and indicate what might be learned from any violation of it. We discuss prospects for improved tests of other relations for isospin-violating effects, including $\Lambda-\Sigma^{0}$ mixing and baryon decuplet mass splittings. We note the inherent limitations in learning individual terms in isospin-violating mass differences.

The stimulus for our re-examination of a 37-year-old problem has come from the prospect for a substantial improvement in the measurement of the $\Xi^{0}$ mass by the $\mathrm{KTeV}$ Collaboration at Fermilab. The present value [6] is $M_{\Xi^{0}}=1314.9 \pm 0.6 \mathrm{MeV}$, while the next most poorly measured mass is $M_{\Xi^{-}}=1321.32 \pm 0.13 \mathrm{MeV}$. It is very likely that $\mathrm{KTeV}$ could measure the $\Xi^{0}$ mass to comparable or better accuracy, perhaps to $\pm 0.1 \mathrm{MeV}$ [7]. At the same time, a new round of experiments with hyperon beams [8, 9] is capable of improving information on $\Xi^{-}$and hyperon resonance masses, while experiments at the Continuous Electron Beam Accelerator Facility (CEBAF) can improve our knowledge of $\Delta$ resonance isospin splittings. The need for such improvement has been stressed recently in Ref. [10].

We begin in Section II with a general discussion of quark-model effects on isospinviolating mass differences, ending up with a derivation of (1) and several other relations. These are the most general which follow from the absence of three-body effects [4]. We discuss the present and potential experimental situation in Section III, and the degree to which it is possible to estimate the individual contributions to mass splittings in Section IV. We remark on sources of possible violation of Eq. (1) and the other relations in Section V, comparing our work with the more general treatment of Ref. [4]. We comment briefly on charmed baryons in Section VI, and summarize in Section VII. 


\section{ISOSPIN VIOLATIONS IN THE QUARK MODEL}

\section{A. Quark mass differences}

The $u$ and $d$ quarks have intrinsic masses which differ by a couple of MeV. Typical values at scales of $1 \mathrm{GeV}$ [11] are $m_{u} \simeq 5 \mathrm{MeV} / c^{2}, m_{d} \simeq 9 \mathrm{MeV} / c^{2}$. Corresponding estimates for the strange quark mass range from about 100 to $200 \mathrm{MeV} / c^{2}$. When quarks are incorporated into hadrons, more appropriate "constituent" values (see, e.g., Refs. [3, 12, 13]) are $m_{u}, m_{d}=\mathcal{O}(350) \mathrm{MeV} / c^{2}, m_{s}=\mathcal{O}(500) \mathrm{MeV} / c^{2}$, with $m_{d}-m_{u}$ of order a few $\mathrm{MeV} / c^{2}$ but quite uncertain. We shall denote the constituentquark isospin-violating mass difference by $\Delta \equiv m_{u}-m_{d}$. It will be a free parameter in our description of isospin-violating baryon mass splittings.

The quarks' kinetic energies $T$ may also depend on their masses. Without detailed knowledge of dynamics, it is difficult to anticipate this dependence. For an effective potential $V=\lambda r^{\nu}$, the virial theorem $\langle T\rangle=\langle(r / 2) d V / d r\rangle$ implies $\langle T\rangle=\lambda \nu\left\langle r^{\nu}\right\rangle / 2$,

while the scaling of the Schrödinger equation 114 implies $\left\langle r^{\nu}\right\rangle \sim m_{Q}^{-\nu /(\nu+2)}$. Thus for a potential with $\nu<0$ kinetic energies increase with increasing quark mass, while for a potential with $\nu>0$ kinetic energies decrease with increasing quark mass. We shall consequently parametrize kinetic energies simply with labels $K_{q}$ for those contributions which act as one-body operators and $K_{q_{i} q_{j}}$ for those contributions which depend on interactions with each individual other quark.

\section{B. Pairwise Coulomb interactions}

Each quark pair in a hadron has a Coulomb interaction energy

$$
\Delta E_{i j \mathrm{em}}=\alpha Q_{i} Q_{j}\left\langle\frac{1}{r_{i j}}\right\rangle,
$$

where $\alpha \simeq 1 / 137$ is the electromagnetic fine structure constant, $Q_{i}$ is the charge of quark $i$ in units of the proton charge, and $\left\langle 1 / r_{i j}\right\rangle$ is the expectation value of the inverse distance between the members of the pair.

In the flavor-SU (3) limit one expects $\left\langle 1 / r_{i j}\right\rangle$ to be universal throughout a multiplet. Thus, for example, every quark pair in every octet baryon should have the same value of this quantity. In this limit, we parametrize the interaction energy $\Delta E_{i j \mathrm{em}}=a Q_{i} Q_{j}$, where $a$ is some universal constant. We shall explore the possible violations of this assumption in Sec. V.

\section{Strong hyperfine interactions}

Quarks are bound in hadrons by a dominantly spin-independent force which becomes strong at large distances. In addition, they experience a spin-dependent force due to gluon exchange which acts dominantly on pairs in an S-wave state. For pairs of quarks in a baryon, one has a (strong) hyperfine interaction energy

$$
\Delta E_{i j \mathrm{HFs}}=\text { const. } \frac{\left|\Psi_{i j}(0)\right|^{2}\left\langle\sigma_{i} \cdot \sigma_{j}\right\rangle}{m_{i} m_{j}},
$$


where $\left|\Psi_{i j}(0)\right|^{2}$ is the square of the S-wave wave function of two quarks at zero relative separation, and the constant is universal for all pairs of quarks in a baryon. In the limit in which the hyperfine interaction is given by one-gluon exchange, this constant is of first order in $\alpha_{s}$. The nucleon $-\Delta$ splitting of about $300 \mathrm{MeV} / c^{2}$ is an example of a QCD hyperfine effect.

We shall assume for the moment that $\left|\Psi_{i j}(0)\right|^{2}$ is universal for all quark pairs in octet baryons. We then find a contribution to the hyperfine energy $\Delta E_{i j} \mathrm{HFs}=$ $b\left\langle\sigma_{i} \cdot \sigma_{j}\right\rangle /\left(m_{i} m_{j}\right)$.

The calculation of strong hyperfine splittings in baryons requires evaluation of $\left\langle\sigma_{i} \cdot \sigma_{j}\right\rangle$ for each quark pair. Since $\mathbf{S}=\sum_{i}\left(\sigma_{i} / 2\right)$, we use the value of $\mathbf{S}^{2}$ to evaluate the sum of $\left\langle\sigma_{i} \cdot \sigma_{j}\right\rangle$ for all pairs, with the result

$$
\left\langle\sum_{i<j} \sigma_{i} \cdot \sigma_{j}\right\rangle=\left\{\begin{array}{ll}
-3 & (S=1 / 2) \\
+3 & (S=3 / 2)
\end{array}\right\} .
$$

In any color-singlet baryon, Fermi statistics and the antisymmetry of any two quarks with respect to color interchange lead to symmetry in the remaining (space $\times$ spin $\times$ flavor) variables. For ground-state baryons with two identical quarks (including those involved in the Coleman-Glashow relation), the two like quarks must hence be in a state symmetric with respect to spin, i.e., of spin 1, and hence must have $\langle\sigma \cdot \sigma\rangle=1$. For any baryon in the flavor decuplet, such as $\Delta^{++}=u u u$ (with $S=3 / 2$ ), each pair has this value, consistent with the result (蛋. For any octet baryon state $q_{i} q_{i} q_{j}(j \neq i)$, one then concludes $\left\langle\sigma_{i} \cdot \sigma_{j}\right\rangle=-2$.

\section{Electromagnetic hyperfine interactions}

The electromagnetic interaction between quarks in a baryon has a spin-dependent (hyperfine) contribution

$$
\Delta E_{i j \mathrm{HFe}}=-\frac{2 \pi \alpha Q_{1} Q_{2}\left|\Psi(0)_{i j}\right|^{2}\left\langle\sigma_{i} \cdot \sigma_{j}\right\rangle}{3 m_{i} m_{j}} .
$$

Again assuming universality of the wave functions, we can parametrize this effect as $\Delta E_{i j \mathrm{HFe}}=c Q_{i} Q_{j}\left\langle\sigma_{i} \cdot \sigma_{j}\right\rangle /\left(m_{i} m_{j}\right)$.

\section{E. Summary of effects}

We can now collect all the results for baryon isospin-violating mass shifts into quantities organized according to the isospin of the splittings. We obtain seven $I=1$ combinations, three $I=2$ combinations, and one $I=3$ combination [4].

1. $\Delta I=1$ splittings.

$$
\begin{gathered}
N_{1} \equiv M_{p}-M_{n}=\Delta+K_{u}-K_{d}+K_{u u}-K_{d d} \\
+\frac{a}{3}+b\left(\frac{1}{m_{u}^{2}}-\frac{1}{m_{d}^{2}}\right)+\frac{c}{9}\left(\frac{4}{m_{u}^{2}}-\frac{1}{m_{d}^{2}}\right)
\end{gathered}
$$




$$
\begin{gathered}
\Sigma_{1} \equiv M_{\Sigma^{+}}-M_{\Sigma^{-}}=2 \Delta+2\left(K_{u}-K_{d}\right)+2\left(K_{u s}-K_{d s}\right)+K_{u u}-K_{d d} \\
-\frac{a}{3}+b\left(\frac{1}{m_{u}^{2}}-\frac{1}{m_{d}^{2}}+\frac{4}{m_{d} m_{s}}-\frac{4}{m_{u} m_{s}}\right)+\frac{c}{9}\left(\frac{4}{m_{u}^{2}}-\frac{1}{m_{d}^{2}}+\frac{4}{m_{d} m_{s}}+\frac{8}{m_{u} m_{s}}\right) \\
\Xi_{1} \equiv M_{\Xi^{0}}-M_{\Xi^{-}}=\Delta+K_{u}-K_{d}+2\left(K_{u s}-K_{d s}\right) \\
-\frac{2 a}{3}+b\left(\frac{4}{m_{d} m_{s}}-\frac{4}{m_{u} m_{s}}\right)+\frac{c}{9}\left(\frac{4}{m_{d} m_{s}}+\frac{8}{m_{u} m_{s}}\right) \\
M_{\Lambda \Sigma^{0}}=\sqrt{3}\left[b\left(\frac{1}{m_{d} m_{s}}-\frac{1}{m_{u} m_{s}}\right)+\frac{c}{9}\left(\frac{1}{m_{d} m_{s}}+\frac{2}{m_{u} m_{s}}\right)\right] \\
\Delta_{1} \equiv 3 M_{\Delta^{++}}+M_{\Delta^{+}}-M_{\Delta^{0}}-3 M_{\Delta^{-}}=10\left[\Delta+K_{u}-K_{d}+K_{u u}-K_{d d}\right. \\
\left.+\frac{a}{3}+b\left(\frac{1}{m_{u}^{2}}-\frac{1}{m_{d}^{2}}\right)+\frac{c}{9}\left(\frac{4}{m_{u}^{2}}-\frac{4}{m_{d}^{2}}\right)\right] \\
\Sigma_{1}^{*} \equiv M_{\Sigma^{*+}}-M_{\Sigma^{*-}}=2 \Delta+2\left(K_{u}-K_{d}\right)+2\left(K_{u s}-K_{d s}\right)+K_{u u}-K_{d d} \\
+b\left(\frac{1}{m_{u}^{2}}-\frac{1}{m_{d}^{2}}+\frac{2}{m_{u} m_{s}}-\frac{2}{m_{d} m_{s}}\right)+\frac{c}{9}\left(\frac{4}{m_{u}^{2}}-\frac{1}{m_{d}^{2}}-\frac{4}{m_{u} m_{s}}-\frac{2}{m_{d} m_{s}}\right) \\
\Xi_{1}^{*} \equiv M_{\Xi^{* 0}}-M_{\Xi^{*-}}=\Delta+K_{u}-K_{d}+2\left(K_{u s}-K_{d s}\right) \\
-\frac{2 a}{3}+2 b\left(\frac{1}{m_{u} m_{s}}-\frac{1}{m_{d} m_{s}}\right)-\frac{c}{9}\left(\frac{2}{m_{d} m_{s}}+\frac{4}{m_{u} m_{s}}\right)
\end{gathered}
$$

These quantities are related to one another by

$$
\begin{gathered}
N_{1}=\Sigma_{1}-\Xi_{1}=\Sigma_{1}^{*}-\Xi_{1}^{*}=\Delta_{1} / 10, \\
2 \sqrt{3} M_{\Lambda \Sigma^{0}}=\Sigma_{1}-\Sigma_{1}^{*} .
\end{gathered}
$$

The Coleman-Glashow relation (1) is one of these; the remaining ones require information on the baryon decuplet. Eq. (14) has been derived in Ref. 15.

2. $\Delta I=2$ splittings.

$$
\begin{gathered}
\Sigma_{2} \equiv M_{\Sigma^{+}}+M_{\Sigma^{-}}-2 M_{\Sigma^{0}}=K_{u u}+K_{d d}-2 K_{u d} \\
+a+b\left(\frac{1}{m_{u}}-\frac{1}{m_{d}}\right)^{2}+\frac{c}{9}\left(\frac{2}{m_{u}}+\frac{1}{m_{d}}\right)^{2}, \\
\Delta_{2} \equiv M_{\Delta^{++}}-M_{\Delta^{+}}-M_{\Delta^{0}}+M_{\Delta^{-}}=2\left[K_{u u}+K_{d d}-2 K_{u d}\right. \\
\left.+a+b\left(\frac{1}{m_{u}}-\frac{1}{m_{d}}\right)^{2}+\frac{c}{9}\left(\frac{2}{m_{u}}+\frac{1}{m_{d}}\right)^{2}\right], \\
\Sigma_{2}^{*} \equiv M_{\Sigma^{*+}}+M_{\Sigma^{*-}}-2 M_{\Sigma^{0}}=K_{u u}+K_{d d}-2 K_{u d} \\
+a+b\left(\frac{1}{m_{u}}-\frac{1}{m_{d}}\right)^{2}+\frac{c}{9}\left(\frac{2}{m_{u}}+\frac{1}{m_{d}}\right)^{2} .
\end{gathered}
$$

These quantities are all proportional to one another:

$$
\Sigma_{2}=\Delta_{2} / 2=\Sigma_{2}^{*}
$$


The $\Delta I=2$ relation (15) will turn out to be useful, when combined with the others, in determining the individual contributions to the mass splittings (Sec. IV).

3. $\Delta I=3$ splitting. One combination of the $\Delta$ masses vanishes:

$$
\Delta_{3} \equiv M_{\Delta^{++}}-3 M_{\Delta^{+}}+3 M_{\Delta^{0}}-M_{\Delta^{-}}=0 .
$$

This will be useful in eliminating the $\Delta^{-}$mass from other relations, since no value is quoted [6] for it. If (19) is used, one finds $M_{\Delta^{+}}-M_{\Delta^{0}}=(1 / 3)\left(M_{\Delta^{++}}-M_{\Delta^{-}}\right)=N_{1}$ and $M_{\Delta^{++}}-2 M_{\Delta^{+}}+M_{\Delta^{0}}=M_{\Delta^{+}}-2 M_{\Delta^{0}}+M_{\Delta^{-}}=\Delta_{2} / 2$. These relations have been employed in many of the studies in Refs. [1], [4], [5], and earlier works quoted by them.

4. Discussion. We did not need to expand in powers of $m_{s}-m_{d}$ or $m_{d}-m_{u}$ to obtain the above relations. On the other hand, we did assume universality of quarkmodel wave functions, i.e., universal values of $\left\langle 1 / r_{i j}\right\rangle$ and $\left|\Psi(0)_{i j}\right|^{2}$. Since the quark masses are arbitrary, the electromagnetic hyperfine terms automatically will have the same structure as the strong ones, aside from a weighting of inverse quark masses by quark charges. As we shall see in Sec. V, one can in fact relax the universality assumption, replacing it by universality of interaction of any given pair regardless of the baryon in which it is found. The two-body kinetic terms in fact exhibit this feature.

The relations for the mass splittings are equivalent, upon identification of terms, to ones which have been obtained previously within the context of specific models [16, 17]. However, as we shall see in Sec. IV, in the present approach one is prevented from identifying the magnitude of individual terms (such as Coulomb and hyperfine self-energies) without making additional assumptions about the one- and two-body kinetic terms. The relations indeed hold under arbitrary forms of one- and two-body quark forces. For spin-independent forces this is illustrated by the completely general nature of the $K_{i}$ and $K_{i j}$ terms, but it is true when spin is included as well. This was, in fact, noted before the advent of QCD [18, 19].

\section{EXPERIMENTAL SITUATION}

\section{A. Present}

The individual masses of members of the baryon octet and decuplet are summarized in Table 1 [6]. The measured values of the octet mass splittings are

$$
\begin{aligned}
& N_{1}=-1.293 \mathrm{MeV} / c^{2}, \quad \Sigma_{1}=-8.07 \pm 0.08 \mathrm{MeV} / c^{2}, \\
& \Xi_{1}=-6.4 \pm 0.6 \mathrm{MeV} / c^{2}, \quad \Sigma_{2}=1.71 \pm 0.18 \mathrm{MeV} / c^{2} .
\end{aligned}
$$

The left-hand side of the relation (1) is $7.7 \pm 0.6 \mathrm{MeV} / c^{2}$, consistent with the righthand side of $8.07 \pm 0.08 \mathrm{MeV} / c^{2}$.

The measured values of the decuplet mass splittings are

$$
\Sigma_{1}^{*}=-4.4 \pm 0.64 \mathrm{MeV} / c^{2} \quad, \quad \Xi_{1}^{*}=-3.2 \pm 0.6 \mathrm{MeV} / c^{2}, \quad \Sigma_{2}^{*}=2.6 \pm 2.1 \mathrm{MeV} / c^{2}
$$


Table 1: Masses of baryon octet and decuplet members, in $\mathrm{MeV} / \mathrm{c}^{2}$.

\begin{tabular}{|cc|cc|}
\hline Octet & & Decuplet & \\
Baryon & Mass & Baryon & Mass \\
\hline$p$ & $938.27231 \pm 0.00028$ & $\Delta^{++}$ & $1231.04 \pm 0.17$ \\
$n$ & $939.56563 \pm 0.00028$ & $\Delta^{+}$ & $1234.9 \pm 1.4$ \\
$\Lambda$ & $1115.684 \pm 0.006$ & $\Delta^{0}$ & $1233.77 \pm 0.19$ \\
$\Sigma^{+}$ & $1189.37 \pm 0.07$ & $\Sigma^{*+}$ & $1382.8 \pm 0.4$ \\
$\Sigma^{0}$ & $1192.55 \pm 0.08$ & $\Sigma^{* 0}$ & $1383.7 \pm 1.0$ \\
$\Sigma^{-}$ & $1197.436 \pm 0.033$ & $\Sigma^{*-}$ & $1387.2 \pm 0.5$ \\
$\Xi^{0}$ & $1314.9 \pm 0.6$ & $\Xi^{* 0}$ & $1531.80 \pm 0.32$ \\
$\Xi^{-}$ & $1321.32 \pm 0.13$ & $\Xi^{*-}$ & $1535.0 \pm 0.6$ \\
\hline
\end{tabular}

The relation

$$
\Sigma_{1}-\Xi_{1}\left(=-1.67 \pm 0.6 \mathrm{MeV} / c^{2}\right)=\Sigma_{1}^{*}-\Xi_{1}^{*}\left(=-1.2 \pm 0.9 \mathrm{MeV} / c^{2}\right)
$$

is satisfied, albeit with large uncertainty. So is the relation

$$
\Sigma_{2}\left(=1.71 \pm 0.18 \mathrm{MeV} / c^{2}\right)=\Sigma_{2}^{*}\left(=2.6 \pm 2.1 \mathrm{MeV} / c^{2}\right) .
$$

In order to compare relations involving $\Delta$ masses, we must use the vanishing of the $\Delta I=3$ combination $\Delta_{3}$ to eliminate $M\left(\Delta^{-}\right)$. We then obtain one relation:

$$
M\left(\Delta^{++}\right)-2 M\left(\Delta^{+}\right)+M\left(\Delta^{0}\right)=\Sigma_{2}^{*}\left(=\Sigma_{2}\right) .
$$

The left-hand side of this expression is $-5.0 \pm 2.8 \mathrm{MeV} / c^{2}$, not particularly consistent with (23). We shall see in Sec. V that the hierarchy [4] of mass relations based on the $1 / N_{c}$ expansion moderates this difficulty by finding Eq. (23) to be no more accurate than $\Sigma_{2}=0$ or $\Sigma_{2}^{*}=0$. On the other hand, Eq. (24) with $\Sigma_{2}^{*}$ on the right-hand side is expected in the $1 / N_{c}$ approach to be better behaved by an order of magnitude. It has been noted in Refs. [10] that the value quoted for $M\left(\Delta^{+}\right)$in Ref. [G] may not be reliable.

If we combine $\Delta_{1}=10 N_{1}$ with $\Delta_{3}=0$, we find $M\left(\Delta^{+}\right)=N_{1}+M\left(\Delta^{0}\right)$. (We shall discuss the accuracy of this relation in Sec. V.) We can then substitute in (24) to find (see also, e.g., 可)

$$
M\left(\Delta^{++}\right)-M\left(\Delta^{0}\right)\left(=-2.7 \pm 0.3 \mathrm{MeV} / c^{2}\right)=\Sigma_{2}^{*}\left(\Sigma_{2}\right)+2 N_{1} .
$$

The right-hand side is $-0.0 \pm 2.1 \mathrm{MeV} / c^{2}$ if we use $\Sigma_{2}^{*}$ (permitted in Ref. (四) and $-0.88 \pm 0.18 \mathrm{MeV} / c^{2}$ if we use $\Sigma_{2}$.

\section{B. Future}

The KTeV Collaboration at Fermilab [7] has obtained a large sample of $\Xi^{0}$ 's in a neutral hyperon beam. The detector is able to observe both charged and neutral 
particles in the decay $\Xi^{0} \rightarrow \pi^{0} \Lambda \rightarrow \pi^{0} p \pi^{-}$. Reasonable prospects exist for reducing the error on $M_{\Xi^{0}}$ to $\pm 0.1 \mathrm{MeV} / c^{2}$.

The next most poorly known mass is that of the $\Xi^{-}$. Experiments in a new charged hyperon beam at Fermilab [8, 9] could reduce the errors on this quantity.

The prospects are thus good for reducing the error on the test of the ColemanGlashow relation (11) by at least a factor of 6 , to $0.1 \mathrm{MeV} / c^{2}$ or less. This is comparable to the accuracy to which the relation is expected to hold, according to the analysis of Ref. [1].

The relation (14) predicts $M\left(\Lambda \Sigma^{0}\right)=-1.06 \pm 0.19 \mathrm{MeV} / c^{2}$. A test requires one to measure the isospin impurity of the $\Lambda$ (or, more difficult, of the $\Sigma^{0}$ ). One conceivable way to do this would be to study the deviations from apparent charge-independence in the decays $\Sigma^{*} \rightarrow \pi \Lambda$, taking careful account of phase-space differences and electromagnetic final-state interactions. One would need to measure the widths of $\Sigma^{* \pm}$ to a percent, beyond present accuracy.

The other relations derived above require reduction of errors on the decuplet

masses. Perhaps the best prospects in this respect involve the combinations $\Sigma_{1,2}^{*}$ and $\Xi_{1}^{*}$, for which improved values could be obtained in charged hyperon beams at Fermilab [8, 9]. Studies of $\gamma p \rightarrow \Delta^{+} \rightarrow \pi^{0} p$, for example at the Continuous-Beam Electron Facility (CEBAF), could in principle reduce the error on $M\left(\Delta^{+}\right)$.

\section{INDIVIDUAL TERMS}

With assumptions about quark masses 12 and kinetic one- and two-body terms $K_{q_{i} q_{j}}$, one can evaluate individual terms in the expressions for the mass splittings, such as the quark mass difference $\Delta=m_{u}-m_{d}$ and the Coulomb and electromagnetic hyperfine terms, in a model-independent way. [In several earlier studies, dynamical models permitted estimates of the magnitude of the kinetic one- and two-body terms [16] and hence of $\Delta$.] If one does not estimate kinetic terms, the number of parameters is too large to permit a model-independent evaluation of individual terms.

Among the seven $\Delta I=1$ splittings noted in Section II, there are four relations, so only three are independent. We may take these as $N_{1}, \Sigma_{1}$, and (for example) $\Sigma_{1}^{*}$. Among the three $\Delta I=2$ splittings there are two relations, so we may take the bestknown one $\left(\Sigma_{2}\right)$ as independent. If we neglect the kinetic two-body terms, we have four experimental quantities with which to determine the five quantities $\Delta, K_{u}-K_{d}$, $a, b$, and $c$, given estimates of the nonstrange quark mass $\bar{m} \equiv\left(m_{u}+m_{d}\right) / 2$ and the strange quark $m_{s}$.

Each of the three $\Delta I=1$ splittings contains the same combination $\Delta+K_{u}-K_{d}$. Thus, if we were not concerned with the individual values of $\Delta$ and $K_{u}-K_{d}$, we could hope to use one of the $\Delta I=1$ splittings to eliminate $\Delta+K_{u}-K_{d}$, leaving three parameters (essentially $a, b \Delta$, and $c$ ) to determine with the help of three experimental numbers.

A fit to overall octet and decuplet masses [12] leads to $\bar{m}=363 \mathrm{MeV} / c^{2}, m_{s}=538$ $\mathrm{MeV} / c^{2}$, and hence $r \equiv \bar{m} / m_{s}=0.675$. The same fit also implies $\beta \equiv b / \bar{m}^{2}=50$ $\mathrm{MeV} / c^{2}$ (as determined, for example, by the splitting between nucleon and $\Delta$ states). Thus we might hope to extract a value of $\Delta$ from $b \Delta$ as determined above, and then 
learn $K_{u}-K_{d}$ as well.

Let us neglect the $u-d$ mass difference in $c$ terms, and note that the $b$ term in $\Sigma_{2}$ is of second order in $\Delta$. Then we may write $\Sigma_{2}=a+\gamma$ and

$$
\begin{gathered}
\Sigma_{1}-2 N_{1}+\frac{2}{3} \Sigma_{2}=-\frac{a}{3}+2 \beta \frac{\Delta}{\bar{m}}(1+2 r)+\gamma(1+4 r) / 3, \\
\Sigma_{1}^{*}-2 N_{1}+\frac{\Sigma_{2}}{3}=-\frac{2 a}{3}+2 \beta \frac{\Delta}{\bar{m}}(1-r)-2 \gamma r / 3,
\end{gathered}
$$

where $\gamma \equiv c / \bar{m}^{2}$. Now we substitute for $a=\Sigma_{2}-\gamma$ in (26) and (27) to find

$$
\Sigma_{1}-2 N_{1}+\Sigma_{2}=2(1+2 r)\left(\frac{\beta \Delta}{\bar{m}}+\frac{\gamma}{3}\right) \quad, \quad \Sigma_{1}^{*}-2 N_{1}+\Sigma_{2}=2(1-r)\left(\frac{\beta \Delta}{\bar{m}}+\frac{\gamma}{3}\right) \text {. }
$$

These two combinations are proportional to one another, so that instead of being able to solve for $\beta \Delta$ and $\gamma$ we actually have another mass relation:

$$
\frac{\Sigma_{1}^{*}-2 N_{1}+\Sigma_{2}}{\Sigma_{1}-2 N_{1}+\Sigma_{2}}=\frac{1-r}{1+2 r}=0.14
$$

where we have used $r=0.675$. In substituting experimental values in the lefthand side of this relation, the error on $\Sigma_{1}^{*}$ may be reduced by averaging the direct measurement $\Sigma_{1}^{*}=-4.4 \pm 0.64 \mathrm{MeV} / c^{2}$ with the value $\Sigma_{1}^{*}=\Xi_{1}^{*}+N_{1}=-4.49 \pm$ $0.6 \mathrm{MeV} / c^{2}$ to obtain $\Sigma_{1}^{*}=-4.46 \pm 0.44 \mathrm{MeV} / c^{2}$. The result for the left-hand side is $0.04 \pm 0.13$. The predicted value for $\Sigma_{1}^{*}$ is $-4.82 \pm 0.15 \mathrm{MeV} / c^{2}$, with the dominant error stemming from $\Sigma_{2}$. The corresponding prediction for $\Xi_{1}^{*}=\Sigma_{1}^{*}-N_{1}$ is $-3.52 \pm 0.15 \mathrm{MeV} / c^{2}$. A violation of this relation could signify (a) the presence of significant three-body interactions, (b) a ratio $r$ different from that quoted above, or (c) the non-negligibility of kinetic two-body terms. If these are restored, both the numerator and denominator of the left-hand side of Eq. (29) involve the combination $2\left(K_{u s}-K_{d s}+K_{d d}-K_{u d}\right)$, which is of order $\Delta \times[\mathrm{SU}(3)$ breaking] and thus is not likely to be appreciable.

If the dependence of the kinetic terms on $\Delta$ could be established, one would have an additional constraint, from which the parameters could be determined. As one example, if one totally neglected both the kinetic one- and two-body mass splittings, one could use $N_{1}, \Sigma_{1}$, and $\Sigma_{2}$ to find $\Delta=-2.57 \mathrm{MeV} / c^{2}, a=3.06 \mathrm{MeV} / c^{2}$, and $\gamma=-1.35 \mathrm{MeV} / c^{2}$. This value of $\Delta$ is to be compared with that obtained [16] by Isgur $\left(-6 \mathrm{MeV} / c^{2}\right)$, Capstick $\left(-4.4 \mathrm{MeV} / c^{2}\right)$, Itoh et al. $\left(-3.8 \mathrm{MeV} / c^{2}\right)$, and Franklin and Lichtenberg $\left(-2.8 \mathrm{MeV} / c^{2}\right)$. (These last authors point out that Isgur's difference of one-body terms including kinetic energies is $-3.0 \mathrm{MeV} / c^{2}$, much closer to their (and our) value of $\Delta$.) Our Coulomb term $a$ and electromagnetic hyperfine term $\gamma$ are rather similar to Capstick's. If the effective potential between light quarks corresponds to a power-law $r^{\nu}$ with $\nu>0$, as is appropriate for light quarks [14], the scaling law mentioned earlier would imply $K_{u}>K_{d}$ for $\Delta<0$, and hence the inequality $\Delta<-2.57 \mathrm{MeV} / c^{2}$, which is satisfied in the models of Ref. [16]. 


\section{SYMMETRY VIOLATIONS AND THEIR HIERARCHIES}

\section{A. Non-universality of wave functions}

We assumed universal values of $\left\langle 1 / r_{i j}\right\rangle$ and $\left|\Psi(0)_{i j}\right|^{2}$ in deriving the quark-model results of Sec. II. We found that hyperfine interactions satisfied our mass relations independently of quark masses, indicating that we never needed to assume equality of the hyperfine interaction between two nonstrange quarks and that between a strange and a nonstrange quark. (The hyperfine interaction between two strange quarks never entered into our discussion of $\Delta I \geq 1$ mass relations.) However, we did have to assume that hyperfine interactions between members of a pair were independent of the environment in which these interactions occurred. This assumption was equivalent to the neglect of three-body effects.

Similarly, we did not have to assume the equality of Coulomb interactions between non-strange and strange quarks, but had to assume that these interactions were independent of the environment in which they took place. To illustrate this, let us consider the $a$ terms in the Coleman-Glashow relation (1). We shall label the $a$ contributions by subscripts indicating the interaction quark pair ( $n$ for a nonstrange quark and $s$ for a strange quark) and by a superscript denoting the particle in which the interaction is taking place. Then the Coulomb contribution to $N_{1}+\Xi_{1}$ is $\left(a_{n n}^{N}-2 a_{n s}^{\Xi}\right) / 3$, while that to $\Sigma_{1}$ is $\left(a_{n n}^{\Sigma}-2 a_{n s}^{\Sigma}\right) / 3$. The only way in which these two terms could differ is if two-body forces depended on their environment, a circumstance equivalent to the presence of three-body effects.

As long as isospin-violating effects are strictly of one-body or two-body nature, all the relations we have derived so far will hold. What would be a likely direction for deviations from this circumstance? In the case of the Coulomb interactions illustrated above, we might expect by considering the relative size of reduced-mass effects that a nonstrange pair in the $\Sigma$ would be more deeply bound than a nonstrange pair in a nucleon, and a nonstrange-strange pair in the $\Xi$ more deeply bound than one in a $\Sigma$. In that case we would expect

$$
a_{n n}^{\Sigma}-2 a_{n s}^{\Sigma}>a_{n n}^{N}-2 a_{n s}^{\Xi},
$$

or $\Sigma_{1}-\left(N_{1}+\Xi_{1}\right)>0$. The central value of this relation is in fact less than zero but with large uncertainty.

A similar ordering of effects holds for the strong hyperfine terms, with two-body terms contributing with the same relative signs as in the Coulomb-interaction example. Thus one expects the same sign of the inequality from these terms. On the other hand, the electromagnetic hyperfine contributions to $N_{1}, \Xi_{1}$, and $\Sigma_{1}$ all turn out to be positive, preventing one from making such an argument. The two-body kinetic terms $K_{q_{i} q_{j}}$ are of indefinite sign unless one interprets them in a specific context, e.g., as reduced-mass effects. Thus if it is ever found that $\Sigma_{1}-\left(N_{1}+\Xi_{1}\right)<0$, a culprit within the quark model may be three-body effects in kinetic terms or in electromagnetic hyperfine interactions. One would have to examine specific models in more detail to see if such effects really were important. 


\section{B. Comparison with $1 / N_{c}$ hierarchy}

Jenkins and Lebed [4] have presented a view of isospin-violating mass splittings, based on a systematic expansion in powers of isospin-breaking and $\mathrm{SU}(3)$-breaking terms and powers of $1 / N_{c}$. It is worth reviewing some of the common points and differences with respect to our approach.

(1) The $1 / N_{c}$ approach is completely general, whereas we are seeking interpretations within the quark model.

(2) Jenkins and Lebed expect the Coleman-Glashow relation to be very good. A reduction of errors on $\Xi_{1}$ by a factor of 2 (to $\pm 0.3 \mathrm{MeV} / c^{2}$ ) should still lead to a relation which is satisfied to about a standard deviation. We are unable to make as quantitative a statement, having not estimated three-body effects.

(3) Within the $1 / N_{c}$ approach, certain relations are expected to hold to better accuracy than others, and the hierarchy does not always agree with that associated with the number of interacting quarks.

In the $1 / N_{c}$ approach the $\Delta I=1$ relations

$$
35\left[N_{1}-\Xi_{1}+2 \sqrt{3} M\left(\Lambda \Sigma^{0}\right)\right]-2\left(\Delta_{1}-3 \Sigma_{1}^{*}-4 \Xi_{1}^{*}\right)=0
$$

and

$$
7\left[N_{1}-\Xi_{1}+2 \sqrt{3} M\left(\Lambda \Sigma^{0}\right)\right]-\left(\Delta_{1}-3 \Sigma_{1}^{*}-4 \Xi_{1}^{*}\right)=0
$$

are both expected to hold with the same accuracy, though the first is based on the suppression of a two-body operator and the second is based on the suppression of a three-body operator. In our approach only the second relation holds. Other relations based on three-body operators, which consequently hold in both approaches, and which are expected to be of comparable accuracy to the first two, are the ColemanGlashow relation $N_{1}-\Sigma_{1}+\Xi_{1}=0$ and

$$
-7 N_{1}-5 \Sigma_{1}+2 \Xi_{1}+6 \sqrt{3} M\left(\Lambda \Sigma^{0}\right)+\Delta_{1}+2 \Sigma_{1}^{*}+\Xi_{1}^{*}=0 .
$$

The (three-body) relation $\Delta_{1}=10\left(\Sigma_{1}^{*}-\Xi_{1}^{*}\right)$ is expected to be better-obeyed by an order of magnitude than the above expressions. Combining only the three-body relations, we obtain our previous $\Delta I=1$ results. Including Eq. (31), we obtain the additional results

$$
N_{1}-\Xi_{1}+2 \sqrt{3} M\left(\Lambda \Sigma^{0}\right)=0, \quad \Delta_{1}-3 \Sigma_{1}^{*}-4 \Xi_{1}^{*}=0 .
$$

When combined with previous results, these imply such relations as

$$
M\left(\Lambda \Sigma^{0}\right)=(2 \sqrt{3})^{-1}\left(\Xi_{1}-N_{1}\right)=-1.47 \pm 0.17 \mathrm{MeV} / c^{2}
$$

(as quoted in Ref. [4]), and, eliminating $M\left(\Lambda \Sigma^{0}\right)$ from the above relation and Eq. (14), $\Sigma_{1}-\Sigma_{1}^{*}=\Xi_{1}-N_{1}$, or, using other relations expected to hold to the same order,

$\Sigma_{1}^{*} / 2\left(=-2.2 \pm 0.32 \mathrm{MeV} / c^{2}\right)=\Xi_{1}^{*}\left(=-3.2 \pm 0.6 \mathrm{MeV} / c^{2}\right)=N_{1}\left(=-1.293 \mathrm{MeV} / c^{2}\right)$. 
The result $\Sigma_{1}^{*} / 2=\Xi_{1}^{*}$, as noted in Ref. 四, is obeyed to $0.04 \pm 0.03 \%$. The definition of accuracy adopted there, which we shall use, involves writing mass relations as (LHS) $=($ RHS $)$, with all terms positive; accuracy is then defined as (LHS - RHS) $/[($ LHS + RHS) $/ 2$. The relation $\Sigma_{1}^{*} / 2=N_{1}$ is obeyed to $-0.06 \pm 0.02 \%$, while $\Xi_{1}^{*}=N_{1}$ holds to $-0.077 \pm 0.024 \%$. All these relations are expected to hold to about $\pm 0.03 \%$. Thus the results involving $N_{1}$ correspond to slightly worse accuracy than expected, but not at a significant level. Improvement of experimental accuracy on $\Sigma_{1}^{*}$ and $\Xi_{1}^{*}$ would be very helpful in testing the predicted hierarchy.

We mentioned previously a relation obtained by combining $\Delta_{1}=10 N_{1}$ with $\Delta_{3}=$ 0 , namely $M\left(\Delta^{+}\right)-M\left(\Delta^{0}\right)=N_{1}$. This relation is expected in Ref. [4] to be good to $\pm 0.03 \%$, while it is observed to $0.11 \pm 0.06 \%$. Improved information on the $\Delta^{+}$mass would be needed to test this result significantly. Another such relation is $M\left(\Delta^{++}-\right.$ $M\left(\Delta^{-}\right)=3 N_{1}$, totally untested at present.

In a study of $\Delta I=2$ relations, the $1 / N_{c}$ hierarchy appears to have more success than our neglect of three-body effects. The two-body relation

$$
35 \Sigma_{2}-2\left(3 \Delta_{2}+\Sigma_{2}^{*}\right)=0
$$

and the three-body relation

$$
7 \Sigma_{2}-\left(3 \Delta_{2}+\Sigma_{2}^{*}\right)=0
$$

are expected to hold to the same order, whereas we obtain only the second in the quark model. The relations are satisfied to $0.10 \pm 0.03 \%$ and $0.11 \pm 0.05 \%$, respectively. [We have used $\Delta_{3}=0$ to eliminate $M\left(\Delta^{-}\right)$. The authors of Ref. [4] obtain slightly different results as a result of different $M(\Delta)$ inputs.] Both approaches obtain the three-body relation $\Delta_{2}=2 \Sigma_{2}^{*}$, which is expected in the $1 / N_{c}$ analysis to be an order of magnitude more accurate than (37) or (38).

Combining (37) and (38), we find that to the same accuracy,

$$
\Sigma_{2}\left(=1.71 \pm 0.18 \mathrm{MeV} / c^{2}\right)=0 \quad, \quad \Delta_{2}=-\Sigma_{2}^{*} / 3 .
$$

The result $\Sigma_{2}=0$ is good to $0.07 \%$. This may serve as a benchmark for the accuracy to which (37) and (38) may be expected to hold. The result $\Delta_{2}=-\Sigma_{2}^{*} / 3$, when combined with $\Delta_{2}=2 \Sigma_{2}^{*}$ (expected to be more accurate), implies $\Delta_{2}=0$ and $\Sigma_{2}^{*}=0$. $\Delta_{2}=0$, when combined with $\Delta_{3}=0$, implies

$$
M\left(\Delta^{++}\right)-2 M\left(\Delta^{+}\right)+M\left(\Delta^{0}\right)\left(=-5.0 \pm 2.8 \mathrm{MeV} / c^{2}\right)=0 \quad,
$$

corresponding to an error of $-0.2 \pm 0.1 \%$. This is slightly better than the corresponding relation (24) in our approach (when we use $\Sigma_{2}$ on the right-hand side.) The predicted relation $\Sigma_{2}^{*}\left(=2.6 \pm 2.1 \mathrm{MeV} / c^{2}\right)=0$ is obeyed to $0.09 \pm 0.08 \%$. The poorly obeyed relation (25) is replaced by

$$
M\left(\Delta^{++}\right)-M\left(\Delta^{0}\right)\left(=-2.7 \pm 0.3 \mathrm{MeV} / c^{2}\right)=2 N_{1}\left(=-2.6 \mathrm{MeV} / c^{2}\right),
$$

obeyed to $-0.005 \pm 0.014 \%$; the expected accuracy, however, is only as good as that for $\Sigma_{2}=0$, i.e., $\pm 0.07 \%$. 
The $1 / N_{c}$ hierarchy suggests that the relation $\Delta_{2}=2 \Sigma_{2}^{*}$ should be a factor $\mathcal{O}\left(\epsilon / N_{c}\right)$ more accurate than (37) or (38), where $\epsilon \sim 1 / 4$ describes $\mathrm{SU}(3)$ breaking. Thus, we could expect it to hold to $\pm 0.01 \%$ (or at worst $\pm 0.02 \%$ using the numerical estimates of Ref. (4)). Using the relation $\Delta_{3}=0$ whose errors are negligible by comparison, we find the ensuing relation

$$
M\left(\Delta^{++}\right)-2 M\left(\Delta^{+}\right)+M\left(\Delta^{0}\right)\left(=-5.0 \pm 2.8 \mathrm{MeV} / c^{2}\right)=\Sigma_{2}^{*}\left(=2.6 \pm 2.1 \mathrm{MeV} / c^{2}\right)
$$

to be satisfied to $-0.15 \pm 0.07 \%$. Reduction of the errors on $M\left(\Delta^{+}\right)$and $M\left(\Sigma^{* 0}\right)$ is necessary to perform an incisive test of this result. If we use the relation $M\left(\Delta^{+}\right)=$ $N_{1}+M\left(\Delta^{0}\right)$, expected to be good to $\pm 0.03 \%$ as mentioned above, we find that $M\left(\Delta^{++}\right)-M\left(\Delta^{0}\right)=\Sigma_{2}^{*}+2 N_{1}$ [Eq. (25) with $\Sigma_{2}^{*}$ ] should be satisfied to $\pm 0.03 \%$, whereas it holds to $-0.05 \pm 0.04 \%$. Here the error is dominated by that of $M\left(\Sigma^{* 0}\right)$. As noted earlier, some of these conclusions will be changed if the $\Delta^{+}$mass quoted in Ref. [6] is found to be in error [10].

\section{CHARMED BARYONS}

Although baryons containing light quarks are the present topic, recent works [20, 21] have noted a serious discrepancy between a $\Delta I=2$ relation between charmed and non-charmed baryons. The assumptions of Sec. II lead to the relation 22]

$$
\Sigma_{c 2}\left(=-2.0 \pm 1.3 \mathrm{MeV} / c^{2}\right)=\Sigma_{2}\left(=1.71 \pm 0.18 \mathrm{MeV} / c^{2}\right),
$$

where $\Sigma_{c 2} \equiv M\left(\Sigma_{c}^{++}\right)-2 M\left(\Sigma_{c}^{+}\right)+M\left(\Sigma_{c}^{0}\right)$. Experimental values are taken from Ref. [6]. As noted by [20], the negative sign of $\Sigma_{c 2}$ is very difficult to understand in the quark model.

One $\Delta I=1$ relation [22] follows from our assumptions:

$$
\Sigma_{c 1}-2 \Xi_{c 1}^{\prime}=\Sigma_{1}^{*}-2 \Xi_{1}^{*}
$$

where $\Sigma_{c 1} \equiv M\left(\Sigma_{c}^{++}\right)-M\left(\Sigma_{c}^{0}\right)=0.8 \pm 0.4 \mathrm{MeV} / c^{2}$ [6] and $\Xi_{c 1}^{\prime} \equiv M\left(\Xi_{c}^{\prime+}\right)-M\left(\Xi_{c}^{\prime 0}\right)=$ $-1.7 \pm 4.6 \mathrm{MeV} / c^{2}$ [23]. (Here $\Xi_{c}^{\prime}$ denotes the state in which the light quarks are in a flavor- and spin-symmetric state.) The large error on the last quantity prevents any test at present.

\section{SUMMARY}

The prospect of improved values of masses for baryons, such as $\Xi^{0}$ in the KTeV Experiment at Fermilab, charged hyperons and perhaps $\Sigma^{*}$ 's and $\Xi^{*}$ 's in other Fermilab experiments, and $\Delta$ 's in high-intensity photoproduction studies, has led us to re-examine predictions for isospin splittings within the assumption of one- or twobody effects within the quark model. We have shown that the Coleman-Glashow relation (1) is expected to be satisfied independently of quark masses within this assumption. A deviation from it would have to be ascribed to three-body effects.

Tests of other relations will require improved knowledge of decuplet isospin splittings. (For notation see Sec. II.) These include the $\Delta I=1$ relations $N_{1}=\Delta_{1} / 10=$ 
$\Sigma_{1}^{*}-\Xi_{1}^{*}$ and $2 \sqrt{3} M\left(\Lambda \Sigma_{0}\right)=\Sigma_{1}-\Sigma_{1}^{*}$, the $\Delta I=2$ relations $\Sigma_{2}=\Sigma_{2}^{*}=\Delta_{2} / 2$, and the relation $\Delta_{3}=0$.

We have discussed the degree to which one can isolate individual contributions to mass splittings, given quark masses obtained in fits to baryon octet and decuplet spectra [12]. A model-independent determination of these parameters is not possible as a result of the presence of one- and two-body kinetic energy terms. Under a restricted set of assumptions one obtains the predictions $\Sigma_{1}^{*}=-4.82 \pm 0.15 \mathrm{MeV} / c^{2}$ and $\Xi_{1}^{*}=-3.52 \pm 0.15 \mathrm{MeV} / \mathrm{c}^{2}$.

With dynamical assumptions, it would be possible to estimate kinetic terms. One could thereby solve for quantities such as the intrinsic constituent-quark mass difference $\Delta=m_{u}-m_{d}$, the Coulomb self-energy, and the electromagnetic hyperfine interaction. Knowledge of $\Delta$ would be useful in evaluating heavy meson decay constants using spin-dependent hyperfine interactions in the $D$ and $D^{*}$ systems [24]. Knowledge of the electromagnetic hyperfine interaction term $\gamma$, providing an estimate of $\left|\Psi_{i j}(0)\right|^{2}$ in Eq. (5), would be useful in calculations of nonleptonic weak decays of hyperons [25] or of proton decay [26].

We have compared our approach with that of a systematic $1 / N_{c}$ expansion 4 , where $N_{c}$ is the number of colors in QCD. The presence of two- and three-body operators of similar order in the $1 / N_{c}$ expansion leads to a hierarchy of mass relations somewhat different from ours. That approach suggests that the Coleman-Glashow relation should be good to about $\pm 0.3 \mathrm{MeV} / c^{2}$ or better. The $1 / N_{c}$ expansion also obtains some $\Delta I=1$ relations which are expected to be as good as the ColemanGlashow relation, such as $\Sigma_{1}^{*} / 2=\Xi_{1}^{*}=N_{1}$. It will be interesting to compare violations of these and several $\Delta I=2$ relations with that of the Coleman-Glashow relation once improved data on decuplet isospin splittings are available.

\section{ACKNOWLEDGMENTS}

I wish to thank A. Dighe, J. Franklin, E. Jenkins, A. Manohar, N. Solomey, E. Swallow, and R. Winston for discussions, and E. Jenkins for a careful reading of the manuscript. This work was performed in part at the Aspen Center for Physics and supported in part by the United States Department of Energy under Grant No. DE FG02 90ER40560. 


\section{References}

[1] A. De Rújula, H. Georgi, and S. L. Glashow, Phys. Rev. D 12, 147 (1975); J. Franklin, Phys. Rev. D 12, 2077 (1975); D. B. Lichtenberg, Phys. Rev. D 14, 1412 (1976); W. Celmaster, Phys. Rev. Lett. 37, 1042 (1976); S. Ono, Nucl. Phys. B107, 522 (1976); A. Le Yaouanc et al., Phys. Lett. 72B, 53 (1977); L.-H. Chan, Phys. Rev. D 15, 2478 (1977); C. Itoh et al., Prog. Theor. Phys. 61, 548 (1979); I. Cohen and H. J. Lipkin, Phys. Lett. 84B, 323 (1979); A. D. Sakharov, Zh. Eksp. Teor. Fiz. 79, 350 (1980) [Sov. Phys. - JETP 52, 175 (1980)]; N. Isgur, Phys. Rev. D 21, 779 (1980); J. Franklin and D. B. Lichtenberg, Phys. Rev. D 25, 1997 (1982); S. Capstick, Phys. Rev. D 36, 2800 (1987); G. J. Stephenson, K. Maltman, and T. Goldman, Phys. Rev. D 43, 860 (1991); L. S. Kisslinger, T. Goldman, and Z. Li, hep-ph/9610312, to appear in Phys. Lett. B; J. Franklin, Phys. Rev. D 53, 564 (1996); K. Varga, M. Genovese, J.-M. Richard, and B. Silvestre-Brac, hep-ph/9712326 (unpublished).

[2] S. Coleman and S. L. Glashow, Phys. Rev. Lett. 6, 423 (1961).

[3] A. De Rújula, H. Georgi, and S. L. Glashow, Ref. [1].

[4] E. Jenkins and R. F. Lebed, Phys. Rev. D 52, 282 (1995).

[5] P. F. Bedaque and M. A. Luty, Phys. Rev. D 54, 2317 (1996).

[6] Particle Data Group, R. M. Barnett et al., Phys. Rev. D 54, 1 (1996).

[7] KTeV Collaboration, Fermilab Experiments E-799 and E-832, N. Solomey, private communication.

[8] SELEX Collaboration, Fermilab Experiment E-781, J. Russ, spokesperson.

[9] Hyper-CP Collaboration, Fermilab Experiment E-871, K. B. Luk, spokesperson.

[10] R. E. Cutkosky, Phys. Rev. C 367, 1993 (;) R. Workman, Phys. Rev. C 56, 1645 (1997).

[11] H. Leutwyler, "Light Quark Masses," Lectures at Cargèse Summer School on Masses of Fundamental Particles, August, 1996, Univ. of Bern report, hepph/9609467, to be published by Plenum Press.

[12] S. Gasiorowicz and J. L. Rosner, Am. J. Phys. 49, 954 (1981).

[13] J. L. Rosner, in Testing the Standard Model (Proceedings of the 1990 Theoretical Advanced Study Institute in Elementary Particle Physics, Boulder, Colorado, 3-27 June 1990), edited by M. Cvetic and P. Langacker (World Scientific, Singapore, 1991), p. 91.

[14] C. Quigg and J. L. Rosner, Phys. Rep. 56, 167 (1979). 
[15] J. Franklin, D. B. Lichtenberg, W. Namgung, and D. Carydas, Phys. Rev. D 24, 2910 (1981).

[16] See, e.g., C. Itoh et al.; N. Isgur; J. Franklin and D. B. Lichtenberg; and S. Capstick, in Ref. [四].

[17] The Coulomb term for the mass difference $M_{\Sigma^{-}}-M_{\Sigma^{0}}$ in Table I of the study by N. Isgur [1] should be $2 \epsilon / 3$, not $\epsilon / 3$.

[18] H. R. Rubinstein, F. Scheck, and R. H. Socolow, Phys. Rev. 154, 1608 (1967).

[19] J. Franklin, Phys. Rev. 172, 1807 (1968).

[20] J. Franklin, Phys. Rev. D 53, 564 (1996).

[21] K. Varga, M. Genovese, J.-M. Richard, and B. Silvestre-Brac, hep-ph/9712326 (unpublished).

[22] J. Franklin, Phys. Rev. D 12, 2077 (1975).

[23] CLEO Collaboration, report CLEO-CONF 97-29, presented by S. C. Timm at European Physical Society Conference, Jerusalem, August, 1997 (unpublished).

[24] J. F. Amundson et al., Phys. Rev. D 47, 305 (1993).

[25] A. Le Yaouanc et al., Ref. [1].

[26] M. B. Gavela et al., Phys. Lett. 98B, 51 (1981); Phys. Rev. D 23, 1580 (1981). 